\title{
Partial splenic embolisation and improvement of hypersplenism before liver transplantation
}

\author{
C Villaizán Pérez, J Román Pérez, J I Bilbao Jaureguízar, L Sierrasesúmaga Ariznavarreta, \\ I Villa-Elízaga
}

\begin{abstract}
A case of partial splenic embolisation (PSE) that was performed in a girl with severe leucothrombocytopenia due to hypersplenism is described. Treatment with PSE is a safe and effective alternative to splenectomy that prevents clinical complications due to haematological disorders and improves hypersplenism before liver transplantation.

(Arch Dis Child 1995; 72: 243-244)
\end{abstract}

Keywords: partial splenic embolisation, liver transplantation.

Liver transplantation is widely accepted as the definitive treatment for end stage liver disease in children. Progressive liver failure associated with biliary atresia represents the major and most common indication of this procedure. ${ }^{1}$ Patients should be evaluated before they develop major deterioration of hepatic synthetic function and serious portal hypertension complications. Hypersplenism, a well known complication of portal hypertension, and its secondary haematological disorders, ${ }^{2}$ may accelerate patient deterioration before liver transplantation. We report the use of partial splenic embolisation (PSE) as an alternative procedure that can control hypersplenism in children who may require liver transplantation. ${ }^{3}$

\section{Case report}

A girl, born at full term, had atresia of the extrahepatic biliary system diagnosed during the second week of life. When she was 3 months old, successful Sawaguchi portoenterostomy was performed. At the age of 9 years she was admitted to our hospital with a view to possible liver transplantation because of progressive liver fibrosis and haematological disorders with a mild tendency to spontaneous petechiae. Physical examination showed an enlarged, hard liver and splenomegaly. Haematological tests showed leucopenia (total white cell count $3.2 \times 10^{9} /$, neutrophils $1 \cdot 2 \times 10^{9} / 1$ ) and thrombocytopenia (platelet count $\left.92 \times 10^{9} / 1\right)$. She had raised serum transaminases (aspartate aminotransferase $113 \mathrm{UI} / \mathrm{l}$, alanine aminotransferase $108 \mathrm{UI} / \mathrm{l}$, $\gamma$-glutamyltransferase $867 \mathrm{UI} / \mathrm{l})$, alkaline phosphatase $(2180 \mathrm{UI} / \mathrm{l})$, and total serum cholesterol $(6.76 \mathrm{mmol} / \mathrm{l})$. Upper gastrointestinal endoscopy revealed varices in the lower third of the oesophagus. Abdominal

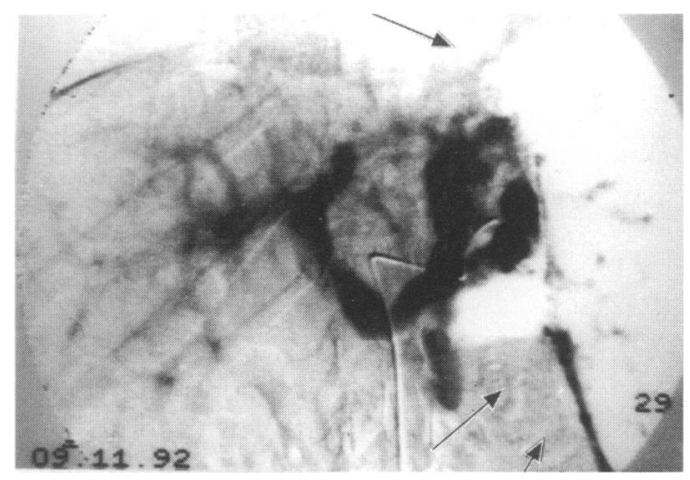

Figure 1 Postembolisation procedure: 70\% of spleen mass has been embolised (arrows).

ultrasound showed hepatomegaly with diffuse distortion of the liver architecture and a $14 \mathrm{~cm}$ splenomegaly; a percutaneous hepatic biopsy specimen showed portal and perilobular fibrosis with minimal ductular proliferation.

Liver transplantation was not considered to be necessary so she was followed up at six to eight month intervals. After four years, platelet and leucocyte counts decreased to $50 \times 10^{9} / 1$ and $2.6 \times 10^{9} / 1$ respectively, splenomegaly and varices increased; however, the clinical symptoms remained unchanged. To avoid severe progressive leucothrombocytopenia and bleeding complications, it was decided to perform PSE. This was performed by the radiology department following the protocol of Spigos et $a l,^{4}$ with a $70 \%$ reduction in the splenic perfusion achieved (fig 1). Peripheral blood count was repeated on days 0,3 , and 7 and subsequently until steady values were

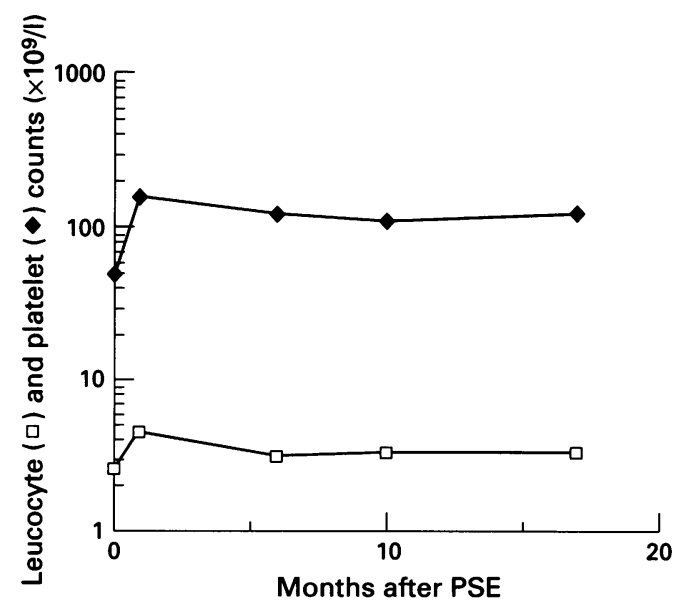

Figure 2 Trends in the leucocyte and platelet counts after $P S E$ (month 0: before PSE). 
reached. Postembolisation complications included moderate pain and low grade fever that had resolved by two days after the procedure.

Platelet and leucocyte counts over time are shown in fig 2. Abdominal ultrasound scans, upper gastrointestinal endoscopy, and liver biopsy performed six months after PSE showed minimal signs of progressive liver disease; the spleen is now smaller. The patient is now asymptomatic and still waiting for liver transplantation.

\section{Discussion}

Extrahepatic biliary atresia consists of atresia or hypoplasia of any portion of the extrahapatic biliary system. An attempt to establish biliary drainage may be made with the hepato-portoenterostomy procedure of Kasai. ${ }^{1}$ This operation is rarely definitive and most children will develop biliary cirrhosis and portal hypertension and finally liver transplantation will be required. These patients often have clinical hypersplenism with low platelet counts that may exacerbate the variceal bleeding. ${ }^{3}$ Treatment with surgical splenectomy carries a high operative risk, has been associated with the occurrence of sepsis, and increases the postoperative morbidity in children who will require liver transplantation. ${ }^{13}$ Since the first patient was treated by Maddison in $1973,{ }^{5}$ PSE has been used in different clinical situ-ations. ${ }^{2}$ With PSE, platelets increase due to the decrease in splenic sequestration; leucocytes initially increase as an acute inflammatory response and decrease in about a week but the total white cell count always remains higher than before embolisation. ${ }^{2}$ Several reports have suggested that PSE is somewhat effective in preventing variceal haemorrhage and childhood hypersplenism can be controlled for at least two years. ${ }^{3}$ In our patient the platelet and leucocyte values have remained above their pre-embolisation levels and no bleeding or infections have occurred. This procedure has the additional advantage of preserving splenic immune function if $30-40 \%$ of splenic perfusion is maintained. ${ }^{2}$ The technique is not exempt from complications because of a decrease in immunological response and the proliferation of anaerobic bacteria. ${ }^{2}$

In conclusion PSE, when performed correctly, is a safe and useful alternative to splenectomy in patients with liver fibrosis and hypersplenism secondary to portal hypertension. Furthermore, it may control hypersplenism before liver transplantation, increasing the likelihood of acquiring a donor organ and making surgical techniques less laborious.

1 Rosenthal P, FACG, Podesta L, Sher L, Makowka L. Liver transplantation in children. Am $\mathcal{F}$ Gastroenterol 1994; 89. 480-92.

2 Bilbao Jaureguizar JI, Sangro B, Longo JM, et al. Splenic embolization prior to myelosuppressive treatment in hepatocarcinoma and active chronic hepatitis. European fournal of Radiology 1992; 15: 211-4.

3 Kumpe DA, Rumack CM, Pretorius DH, Stoecker TJ, Stellin GP. Partial splenic embolization in children with hypersplenism. Radiology 1985; 155: 357-62.

4 Spigos DG, Jonasson O, Mozes M, Capek V. Partial splenic embolization in the treatment of hypersplenism. AfR 1979; 132: 777-82.

5 Maddison F. Embolic therapy of hypersplenism. Invest Radiol 1973; 8: $280-3$. 